\title{
Belgeo
}

Revue belge de géographie

\section{Des échelles pensées pour les enfants aux représentations spatiales produites par les enfants : enjeux politiques des imaginaires géographiques enfantins}

From scales designed for children to spatial representations produced by

children: the political stakes of children's geographical imaginaries

\section{Anne-Cécile Ott}

\section{(2) OpenEdition}

\section{Journals}

\section{Édition électronique}

URL : http://journals.openedition.org/belgeo/19149

DOI : 10.4000/belgeo.19149

ISSN : 2294-9135

Éditeur :

National Committee of Geography of Belgium, Société Royale Belge de Géographie

\section{Référence électronique}

Anne-Cécile Ott, «Des échelles pensées pour les enfants aux représentations spatiales produites par les enfants : enjeux politiques des imaginaires géographiques enfantins », Belgeo [En ligne], 2-3 | 2017, mis en ligne le 31 décembre 2016, consulté le 22 avril 2019. URL : http://journals.openedition.org/ belgeo/19149; DOI : 10.4000/belgeo.19149

Ce document a été généré automatiquement le 22 avril 2019.

Belgeo est mis à disposition selon les termes de la licence Creative Commons Attribution 4.0 International. 


\section{Des échelles pensées pour les enfants aux représentations spatiales produites par les enfants : enjeux politiques des imaginaires géographiques enfantins}

From scales designed for children to spatial representations produced by children: the political stakes of children's geographical imaginaries

\section{Anne-Cécile Ott}

Je remercie Chrystel Oloukoï, Romain Talamoni et Rémi Vergnaud pour leur relecture et leurs conseils.

1 L'enseignement de la géographie, comme celui de l'histoire, attire particulièrement l'attention des didacticiens et des pouvoirs publics car il est stratégique (Bretennot, Mendibil, Rosemberg, 2011) et porteur d'« enjeux civiques et identitaires » (Allieu-Mary, 2009). Il présente des finalités intellectuelles, mais également culturelles et patrimoniales, et donc des enjeux civiques.

2 Il semblerait toutefois que " "l'amont" de l'acte éducatif [soit] mieux connu que son "aval" " (Pingué, 2004). Autrement dit, les chercheurs se sont davantage penchés sur les programmes, les manuels ou autres supports pédagogiques, que sur les pratiques effectives dans les salles de classe ou sur les représentations et pratiques spatiales des enfants eux-mêmes. Par ailleurs, lorsque les rapports enfantins aux espaces sont étudiés, ce sont presque toujours les mêmes territoires qui sont pris en compte, à savoir des espaces de grande échelle, tels que la ville ou le quartier. Les travaux sur les rapports des enfants à la petite échelle sont en effet quasiment inexistants malgré les appels de scientifiques influents dans le domaine (Ansell, 2009; Holt, 2011 ; Philo, 2000 ; LehmanFrisch, Vivet, 2011). 
Ce repli de la recherche sur les échelles micro se retrouve également dans les directives et les pratiques d'enseignement. Cependant, les travaux sur le terrain, auprès des enfants, montrent que ces derniers mobilisent dans leurs imaginaires géographiques (par ailleurs différenciés) des espaces d'échelles très variées, qui vont du (micro)-local au global. Il va alors s'agir de s'interroger sur les raisons de ce décalage, à partir de mes expériences de terrain dans des écoles maternelles et primaires alsaciennes contrastées en termes de public d'élèves ${ }^{1}$, d'analyses des programmes nationaux, des manuels scolaires et des objets géographiques à disposition des enfants, ou encore d'entretiens menés avec le personnel enseignant et des parents d'élèves.

Le cœur du dispositif méthodologique a toutefois consisté en un ensemble d'activités expérimentales construites pour appréhender le mieux possible les représentations et pratiques spatiales enfantines. Avec les enfants de maternelle, ont été menés une activité de dessin commenté, des jeux de sociétés sur les espaces et sociétés du Monde ou de la manipulation d'objets géographiques. Les enfants de CM1-CM2 ont quant à eux fait une activité de brainstorming (durant laquelle ils étaient invités à s'exprimer lors de ma toute première intervention sur ce que leur évoquait la notion d' "Europe»), une activité de photo-elicitation, de listage d'images et de mots représentant plus ou moins l'Europe pour eux ; ils ont également manipulé des objets géographiques (globe, cartes, livres) et discuté autour de ces objets et notamment des représentations de l'Europe qu'ils véhiculaient ${ }^{2}$.

Après avoir abordé la question du repli sur les grandes échelles (principalement nationale et locale) que l'on retrouve dans l'enseignement primaire de la géographie, nous verrons que les enfants sont toutefois toujours confrontés, que ce soit directement ou indirectement, à des échelles beaucoup plus petites. Derrière ce décalage, il semble que se cache un enjeu politique (avec d'une part une dépolitisation institutionnelle, et de l'autre une certaine repolitisation enfantine des représentations des territoires), qu'il s'agira d'expliquer.

\section{L'enseignement primaire de la géographie : un repli sur les grandes échelles}

6 L'enseignement primaire de la géographie est fait de choix : choix des thématiques à étudier, du temps à accorder à tel thème, mais aussi des espaces et territoires à aborder. L'analyse des différents programmes officiels français de ces dernières années nous montre que les directives se concentrent essentiellement sur les espaces de grandes échelles. L'expérience de terrain au sein de classes de maternelle et de primaire (en 2014 et 2015) indique en outre que les pratiques enseignantes semblent également favoriser ce repli sur les échelles nationale et locale.

\section{Une concentration sur les échelles nationale et locale}

\section{Un regard franco-centré}

7 D'après une étude diachronique des derniers programmes et directives ${ }^{3}$ destinés au cycle 3 (qui correspondait encore aux classes de $\mathrm{CM} 1-\mathrm{CM} 2^{4}$ ), on observe une concentration croissante sur le territoire national. Si en 2002, on pouvait lire que "le programme, centré sur l'espace national, est organisé selon trois entrées: le monde, l'Europe et la France », les deux premiers territoires évoquées, de petite échelle, vont ensuite cesser d'être des « entrées » 
pour être davantage des espaces "contextes " servant à éclairer tel ou tel aspect de l'espace national. Les textes officiels indiquent ainsi en 2008 qu'il s'agit maintenant d'aborder « les principales caractéristiques de la géographie de la France dans un cadre européen et mondial ", "le territoire français dans l'Union européenne » ou encore «les Français dans le contexte européen ". Ce sont là les seules mentions d'espaces de petites échelles qui sont faites. Les directives pour le nouveau programme de 2015 évoquent encore moins ces espaces: sur l'ensemble des repères et des thématiques, ils ne sont mentionnés qu'une seule fois, dans le cadre du thème "se déplacer ": "se déplacer de ville en ville, en France, en Europe et dans le monde ", et qu'une seule fois également dans les démarches et contenus d'enseignement (il est précisé que «le(s) lieu(x) de vie de l'élève est(sont) inséré(s) dans des territoires plus vastes, région, France, Europe, monde »). Analysant une interview de 2004 de Philippe Joutard, un responsable des programmes, Géraldine Bozec note bien que «le privilège du national dans les programmes du primaire repose sur [...] une volonté politique constante » (Bozec, 2010, p. 164).

Du côté des pratiques enseignantes, cette focalisation sur le territoire de l'Etat-Nation semblait aussi de mise. Ainsi, lors d'entretiens menés avec les trois enseignants des classes de CM1 ou de CM2 enquêtées en 2015, j'ai pu observer que c'est avant tout la France qui concentre leur attention, bien qu'ils essayent de ne pas négliger l'enseignement de l'Europe, tout de même prévu dans les programmes. L'enseignant de CM1 de l'Ecole C' (qui chaque année suit ses CM1 l'année suivante en CM2) «travaille en général, au CM1, plus autour de l'Europe et au CM2, reste plus sur la France ». J'ai toutefois pu observer qu'avec les CM1, au mois de mars, il venait tout juste de commencer à parler de l'Europe, à partir du remplissage d'un fond de carte avec le nom de certains pays et de certains massifs montagneux. Dans les deux autres classes, à la même période de l'année, seule la France avait été abordée pour l'instant, et les professeurs n'avaient prévu d'aborder l'Europe que de manière allusive : «Alors on va faire la géographie de la France, [...] et après, plus globalement, on va voir sur l'Europe, mais c'est surtout placer l'Europe dans le Monde » (enseignant de la classe de CM2 de l'Ecole B') ; «J'avais prévu de faire la France et l'Europe [...] j'insisterai juste sur le fait que l'Europe existe et que la France en fait partie... » (enseignante de la classe de CM1 de l'Ecole A'). Ainsi, le poids du territoire national semble également indéniable lorsque l'on s'intéresse aux activités concrètes menées au sein des classes.

Il a toutefois été possible de nuancer légèrement ce caractère franco-centré de l'enseignement primaire de la géographie chez les enseignants rencontrés. En effet, mon terrain ayant été effectué en Alsace, région frontalière, marquée par une forte identité, j'ai pu observer une ouverture à une échelle supra-nationale, avec un accent mis notamment sur les relations franco-allemande ou sur la Regio ${ }^{5}$. Dans la classe de l'école B', une intervenante suisse était d'ailleurs venue faire une présentation sur ce territoire singulier.

\section{Le poids du local}

10 Autre échelle qui semble avoir les faveurs des institutions : le local. En maternelle, il s'agit même de la seule échelle abordée par les directives officielles. On peut même parler ici de niveau micro-local, puisque seul «l'environnement immédiat » des enfants est mentionné, comme on peut le lire dans le Bulletin officiel de 2008 :

«Tout au long de l'école maternelle, les enfants apprennent à se déplacer dans

l'espace de l'école et son environnement immédiat. Ils parviennent à se situer par

rapport à des objets ou à d'autres personnes, à situer des objets ou des personnes 
les uns par rapport aux autres ou par rapport à d'autres repères, ce qui suppose une décentration pour adopter un autre point de vue que le sien propre ».

11 Il faut même attendre les classes de CP-CE1 pour voir apparaitre des espaces tel que le quartier : " les élèves découvrent et commencent [alors] à élaborer des représentations simples de l'espace familier : la classe, l'école, le quartier, le village, la ville ».

De plus, alors qu'au cycle 3, ce sont les échelles nationale et européenne qui sont officiellement mises en lumière, l'analyse des pratiques enseignantes montrent que le local occupe toujours une place importante dans l'enseignement de la géographie. Par exemple, les enseignants des classes enquêtées trouvaient encore très important d'insister sur les espaces de la ville ou du quartier. Dans la classe A', les élèves ont ainsi participé à un projet appelé L'Aventure citoyenne, durant lequel ils ont notamment travaillé sur leur quartier, son histoire et sa géographie. L'enseignant de l'école B' interrogé trouvait lui aussi très important d'en revenir à ces bases plus locales :

«Ce qu'on fait souvent c'est la ville, une exploration de la ville, de l'environnement. C'est beau de partir sur du très lointain, sur des grands concepts, mais ça reste conceptuel, alors qu'une exploration du quartier, de la ville... ça c'est quelque chose qu'on fera en fin d'année. On est dans un quartier périphérique, et donc on ira faire une visite du quartier historique de la ville, du centre-ville, sur le terrain. »

\section{Les raisons de ce repli : entre contraintes temporelles et souci de simplicité ?}

13 Le poids des grandes échelles dans l'enseignement primaire français de la géographie, que ce soit du côté des directives institutionnelles ou des pratiques enseignantes, semble pouvoir s'expliquer par plusieurs facteurs.

\section{Un manque de temps}

14 L'explication qui revient systématiquement dans les discours des professeurs enquêtés est le manque de temps auquel ils doivent faire face. Les programmes sont bien souvent jugés trop denses et dès lors, les enseignants sont amenés à faire des choix. Ce qui revient en pratique à se concentrer davantage sur les espaces de grande échelle, supposés davantage connus des enfants et plus importants à maîtriser. Quel que soit le profil social de l'école et de la classe, cet argument était omniprésent, comme l'illustre l'extrait d'entretien cidessous :

«Nous avançons trop lentement, tout prend du temps, l'analyse de document,

l'écriture de la leçon... les enseignants ne finissent jamais le programme alors on

reprend des choses du programme de CE2 » (Enseignant de la classe A').

Il faut également noter qu'en plus des échelles supra-nationales, c'est parfois la discipline dans son ensemble qui peut pâtir du manque de temps ressenti par les enseignants. Le volume horaire censé être dévolu à l'enseignement de la géographie n'était respecté que dans une seule des classes, la classe A' dont l'enseignante était titulaire d'une licence de géographie. L'enseignant de la classe B' a par exemple clairement affirmé qu'il préférait avant tout privilégier le français, les mathématiques et l'allemand.

\section{Un problème de complexité...}

Bien que le manque de temps soit généralement l'explication la plus avancée, la complexité de parler des espaces de petite échelle aux élèves a également été mise en 
avant par les enseignants. Pour eux, parler d'un territoire comme l'Europe à des enfants de 9-10 ans semble trop abstrait ou trop conceptuel. Ainsi, selon l'enseignante de la classe A', au profil plus défavorisé, " parler de l'Europe et de la mondialisation en CM est difficile, [car] les programmes rentrent beaucoup trop dans les détails [...] », et même pour le professeur de la classe C', pourtant d'un très bon niveau, l'Europe "c'est un concept qui reste quand même très abstrait [...], très flou, très, très vague ».

Cette peur de la complexité constatée sur le terrain semble être une constante dans l'enseignement français depuis plusieurs décennies, et notamment depuis la massification scolaire et l'avènement de l'école unique (Terrail, 2016). Quel que soit le niveau d'études considéré, les enquêtes internationales " ont toujours conduit aux mêmes conclusions » :

« Face à des élèves réputés faibles, les contenus des savoirs à enseigner sont jugés trop ambitieux, trop intellectuels, trop abstraits ou trop arides, et l'on préfère penser qu'ils comprendront mieux avec des exemples » (ibid., p. 44).

Cela s'est confirmé dans le discours et les pratiques de l'enseignante du CM1 enquêté de l'école A', qui avait à charge des élèves relativement difficiles: " on se rend compte des lacunes des élèves, on préfere parfois leur enseigner des choses toutes simples plutôt que le contenu des programmes parfois bien loin de leurs préoccupations et impossible à enregistrer pour eux ». Elle préférait ainsi mettre l'accent sur des espaces de grande échelle, ceux du quotidien des enfants: lors de mon terrain, la classe a par exemple travaillé sur les différents commerces et institutions que l'on peut trouver dans une ville, ce qui n'est plus censé être au programme du cycle 3. Comme le dit Jean-Pierre Terrail, les professeurs semblent privilégier le recours aux exemples, mais en géographie la simplification, au cœur des nouveaux paradigmes pédagogiques, passe également par un repli sur les territoires de grande échelle, voire sur le (micro)-local.

19 Toutefois, les professeurs ayant affaire à des élèves en difficulté ne sont pas les seuls à préférer simplifier le contenu des programmes, puisque l'enseignant de la classe C' de CM1 bilingue, avec des élèves d'un très bon niveau général, tenait des propos similaires, et adaptait également son enseignement dans ce sens. Il ne souhaitait par exemple pas aborder la question des institutions européennes, même de manière très large, car « [à cet âge-là], ils mélangent beaucoup, par exemple pour eux, tout ceux qui sont dans l'Union européenne vont avoir l'euro comme monnaie, etc.». Lors des activités menées avec les élèves, j'ai néanmoins pu remarquer que de nombreux enfants de la classe avaient au contraire des connaissances relativement pointues sur ces questions. À l'occasion d'un brainstorming en classe entière sur ce qu'évoquait l'Europe pour les enfants, certains ont par exemple spontanément affirmé qu' "en Europe, on n'utilise pas tous la même monnaie, en Suisse on utilise le franc suisse et en Angleterre, la livre " (Marin*6, 9 ans). D'autres enfants n'hésitaient en outre pas à corriger un camarade qui disait que la Suisse ne faisait pas partie de l'Europe : «si de l'Europe mais pas de l'Union européenne » (Emmanuel*', 9 ans).

\section{Mais des imaginaires géographiques enfantins qui se déploient de l'échelle locale à l'échelle mondiale}

20 Travailler sur le terrain au contact des enfants et mener des activités avec eux permet de confronter les représentations spatiales qu'on leur présente au cours de leur scolarité primaire et celles qui peuplent leurs propres imaginaires géographiques. Or cette 
confrontation semble mettre en évidence un décalage, les représentations spatiales enfantines étant loin de se limiter aux grandes échelles.

\section{Des enfants toujours confrontés (directement et indirectement) aux petites échelles}

Le terrain a donc montré que certains professeurs ont tendance à sous-estimer les savoirs ou les connaissances des enfants. La littérature confirme par ailleurs que cela n'est pas une spécificité de ce terrain d'enquête, mais une constante dans le monde enseignant, principalement en ce qui concerne la géographie et les territoires de grande échelle. Géraldine Bozec, spécialiste de sciences politiques, affirme par exemple que "les enfants ont plus de connaissances sur l'Europe que semblent le supposer les enseignants» (Bozec, 2010, p. 177)7. L'idée que l'imaginaire géographique des enfants ne pourrait s'étendre qu'aux espaces proches et vécus au quotidien est donc répandue, mais les enquêtes et activités menées auprès des enfants ont montré que ce n'était pas le cas.

\section{Par l'expérience des mobilités}

Notons tout d'abord que les enfants sont toujours déjà confrontés aux espaces de plus petite échelle, que ce soit directement (à travers les mobilités) ou indirectement (au moyen des médias par exemple). Leurs imaginaires géographiques ne s'arrêtent pas au local ou à leurs territoires du quotidien (Ansell, 2009).

Si l'on prend l'exemple des mobilités, qu'elles soient touristiques et/ou migratoires, on se rend compte que dès leur plus jeune âge les enfants peuvent avoir fait l'expérience de territoires plus lointains et plus vastes que celui de leur quartier ou de leur ville. Ainsi, dans la classe favorisée de maternelle (classe C), un seul élève n'avait jamais été hors de France, alors que sur 18 cas étudiés en détails ${ }^{8} 17$ pays étrangers ont été parcouru (figure 1). Mais même dans la classe la moins favorisée, on retrouvait des enfants qui avaient parfois beaucoup voyagé, ainsi pour les cas de 13 enfants étudiés en détail, 9 Etats ont été visités (figure 2). 
Figure 1. Dès la maternelle, des enfants qui ont beaucoup voyagé, surtout dans les milieux favorisés...

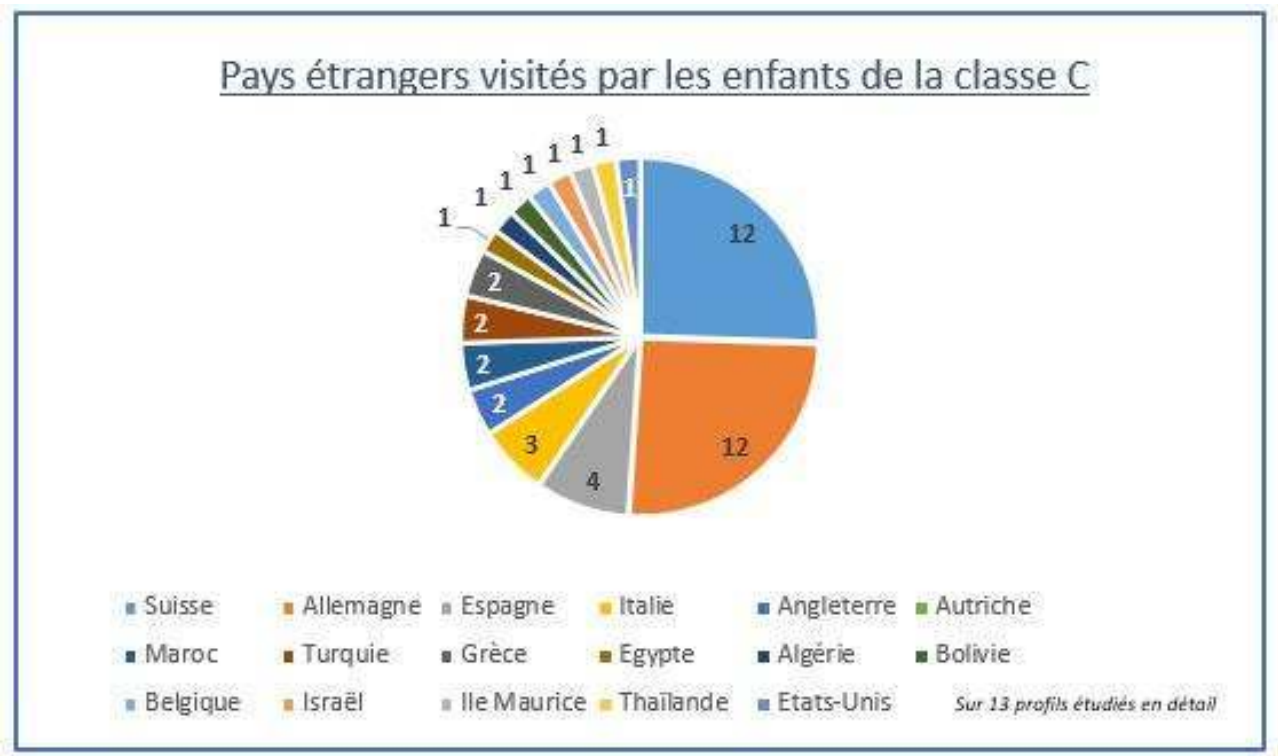

Source : (c) A-C Ott, 2016

Figure 2. ... mais pas uniquement.

\section{Pays étrangers visités par les enfants de la classe A}

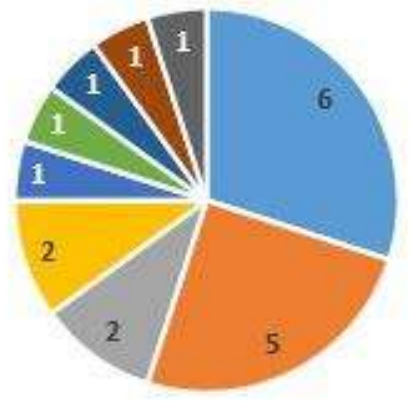

\begin{tabular}{|c|c|c|c|}
\hline \# Turquie & = Allemagne & II Suisse & \\
\hline = Turquie & - Maroc & algérie & \\
\hline - Portugal & - Italie & - Pays-Bas & Sur 13 profils étudiés en détail \\
\hline
\end{tabular}

Source : (C) A-C Ott, 2016

On voit donc qu'une majorité d'enfants, quel que soit leur profil, avait déjà une expérience d'espaces variés, ne se limitant pas à des espaces de proximité, et ces pratiques ont semblé joué un rôle important sur leurs représentations, comprises comme « un ensemble de savoirs et de croyances portant sur des objets ou des concepts » (Beauguitte, Didelon-Loiseau, 2013). 


\section{Par la confrontation aux médias}

Les médias sont également une source d'information capitale qui permet de confronter les enfants à des territoires d'échelles variées : « la télévision et les autres médias confrontent les enfants au "dehors" "(Aitken et Hermann, 1997, p. 81). Ainsi même un enfant qui n'aurait jamais quitté les frontières de la France, est a priori toujours confronté, ne seraitce que de manière indirecte, à ces espaces plus lointains, par les différents médias auxquels il a accès (Ansell, 2009; Valentine et Holloway, 2001, 2002). Et ce de plus en plus à l'ère du numérique : beaucoup d'enfants, de milieux divers, me parlaient de leurs tablettes, ou des tablettes de leurs parents, ou encore d'informations qu'ils avaient entendues sur Internet, à la télévision ou à la radio. L'influence de ces médias serait en croissance et dès lors «le discours scolaire interfere de plus en plus avec des sources d'informations concurrentes: presse, TV, cinéma, bande dessinée, jeux vidéo, etc. » (Brennetot et Rosemberg, 2013) ; il est donc important de ne pas négliger ces autres sources. Le terrain a d'ailleurs confirmé à plusieurs reprises l'influence des médias sur les représentations spatiales et imaginaires géographiques des enfants. En maternelle, ces derniers faisaient souvent référence à des films ou albums illustrés, qui étaient une de leurs principales sources de (re)connaissance des espaces. De même, ces supports jouaient un rôle important sur les espaces aimés par les acteurs rencontrés. C'est ce dont témoignent les extraits suivants de carnet de terrain :

Antoine* (5 ans, classe C) : Oh la statue de la liberté !!

Jean* (5 ans, classe C) : New York!

Antoine* : C'est New York!

Moi : Comment vous connaissez ça ? La statue de la liberté et New York?

Antoine* : Dans Moi, Moche et Méchant on l'a vu.

Benjamin*: J'aime bien l'Afrique. Moi j'ai Kirikou et les bêtes sauvages, Kirikou et la sorcière, Kirikou les hommes et les femmes...

Pauline*[montrant l'Afrique sur un planisphère]: J'aime ça parce que j'aime Kirikou.

\section{Les exemples des représentations du Monde et de l'Europe}

Les enfants ont donc bien des représentations et des pratiques d'autres espaces que ceux de proximité. Le terrain montre néanmoins qu'il est vrai que les espaces de grande échelle peuvent rester difficiles à appréhender pour certains enfants. Lors d'une activité de dessin commenté où je demandais à des élèves de grande section de maternelle de me "dessiner le Monde ", " ce qu'ils avaient dans la tête quand on leur disait "le Monde" ", j'ai pu voir la surprise ou la perplexité dans les yeux de certains d'entre eux. Quelques enfants restaient devant leur feuille sans bouger. Je les interrogeais alors et s'en suivaient parfois des dialogues surprenants comme celui que j'ai eu avec Héloïse* (5 ans, classe C) :

Heloisse : Mais je ne pense pas à un monde moi.

Moi : Tu penses à quoi ?

Héloïse* : À mon doudou.

Chez les plus grands également, des confusions pouvaient régner : ainsi, il n'était pas évident pour tous les élèves de mettre une réalité derrière la notion d'Europe. Pour Kévin* (10 ans, classe A') par exemple, l'Europe était un espace qui s'étendait sur l'ensemble des continents européen, asiatique et africain (figure 3). 
Figure 3. Une vision très large de l'Europe.

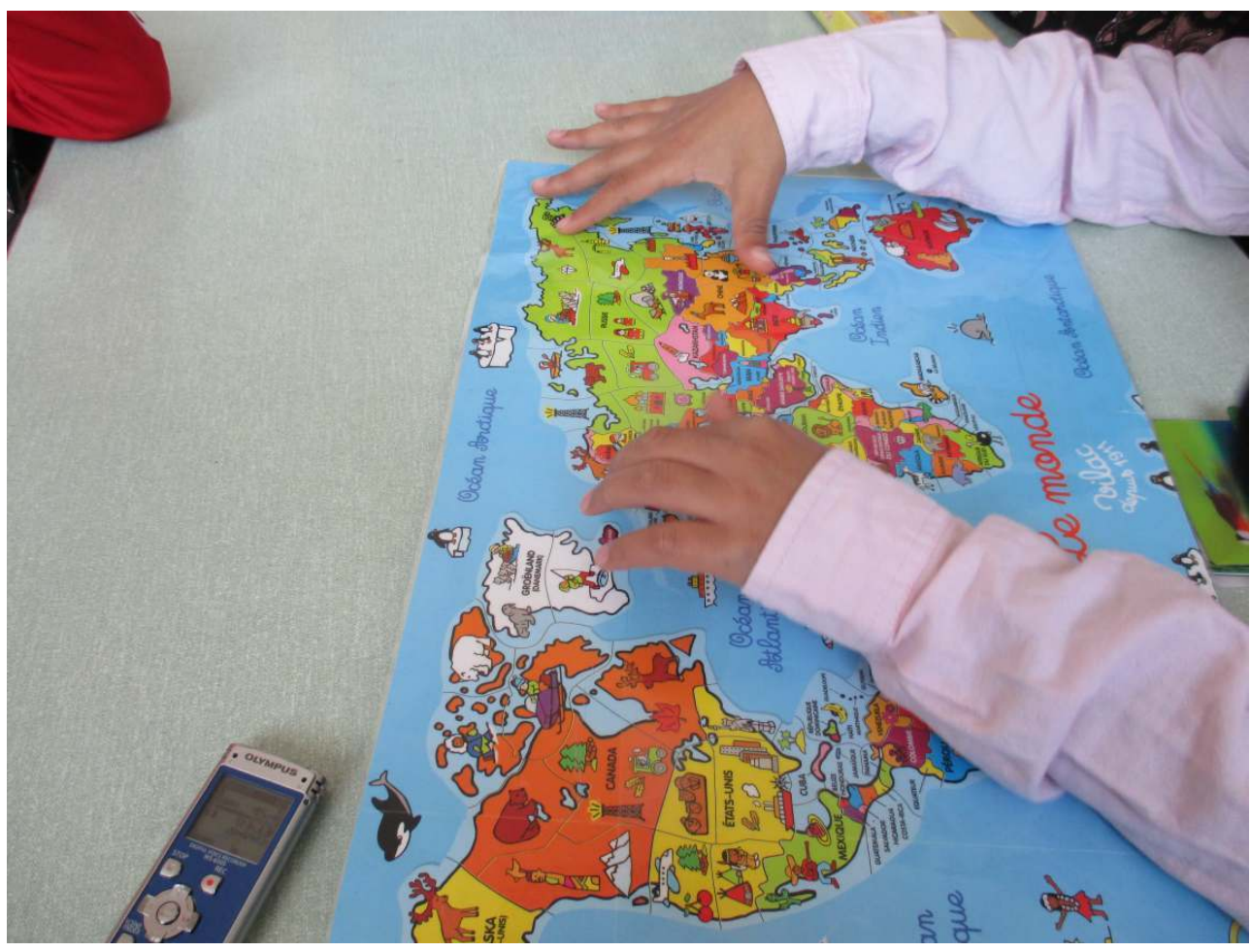

Source : ( ) A-C Ott, 2015

28 Et le territoire national semblait effectivement occuper une place primordiale dans les imaginaires géographiques des enfants enquêtés, qu'il s'agisse de la France et/ou de leur pays d'origine ou de celui de leur famille. Lors des ateliers de manipulations de cartes, de globe ou d'atlas que je menais en petits groupes, les élèves cherchaient avant tout à situer leur pays d'appartenance.

Cela ne veut pourtant pas dire que des espaces de petite échelle comme l'Europe ou le Monde étaient absents de leurs imaginaires géographiques. Au contraire, dès le plus jeune âge, les enfants produisaient des représentations de ces espaces, et parfois même des représentations pointues et techniques. C'est ainsi que quelques élèves de maternelle ont produit lors de l'activité de dessin commenté, une vision globale (figure 4), voire cartographique du Monde (figure 5). 
Figure 4. « Le Monde c'est la planète » (Alexandre, 5 ans, Classe C).

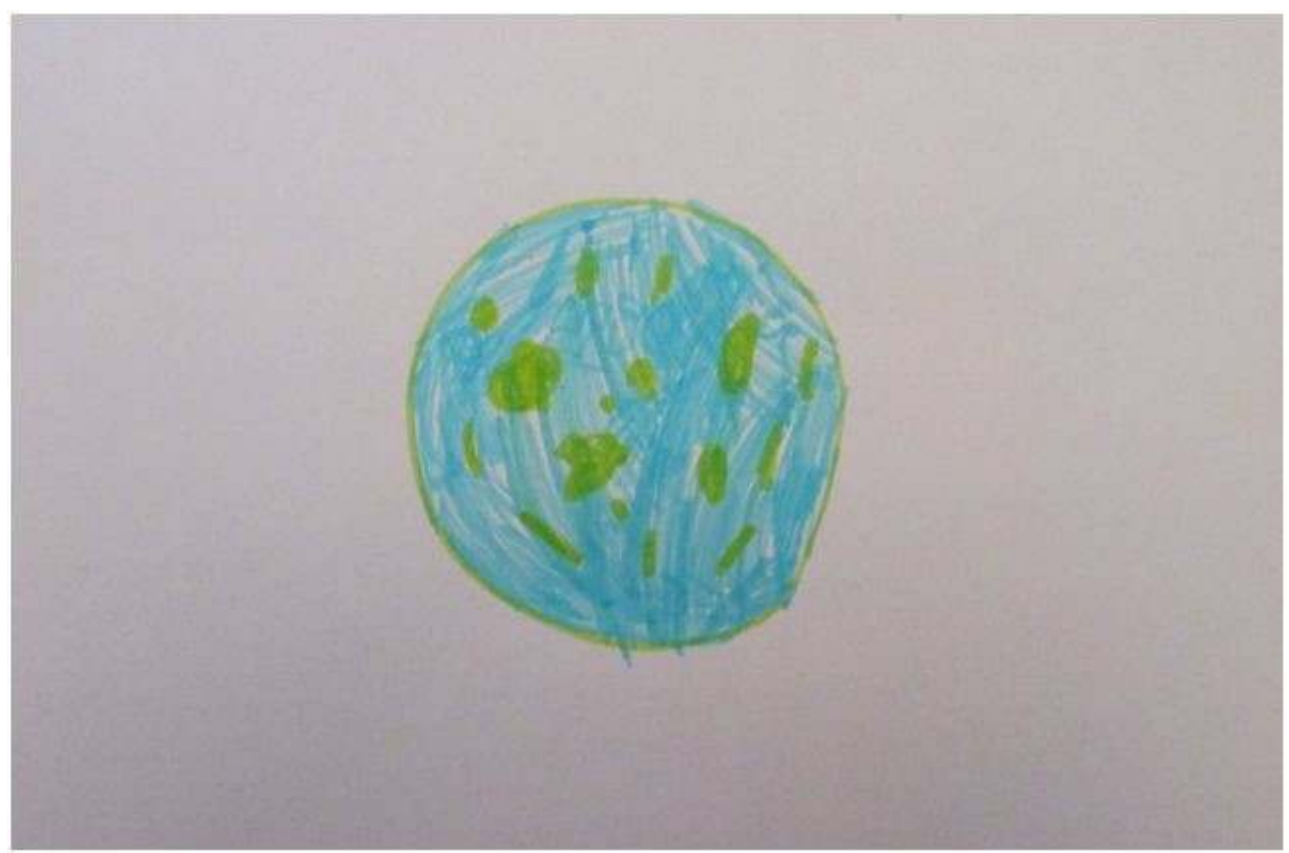

Source : ( ) A.-C. Ott, 2014

Figure 5. Le Monde vu comme un planisphère (Raphaël, 5 ans, classe C).

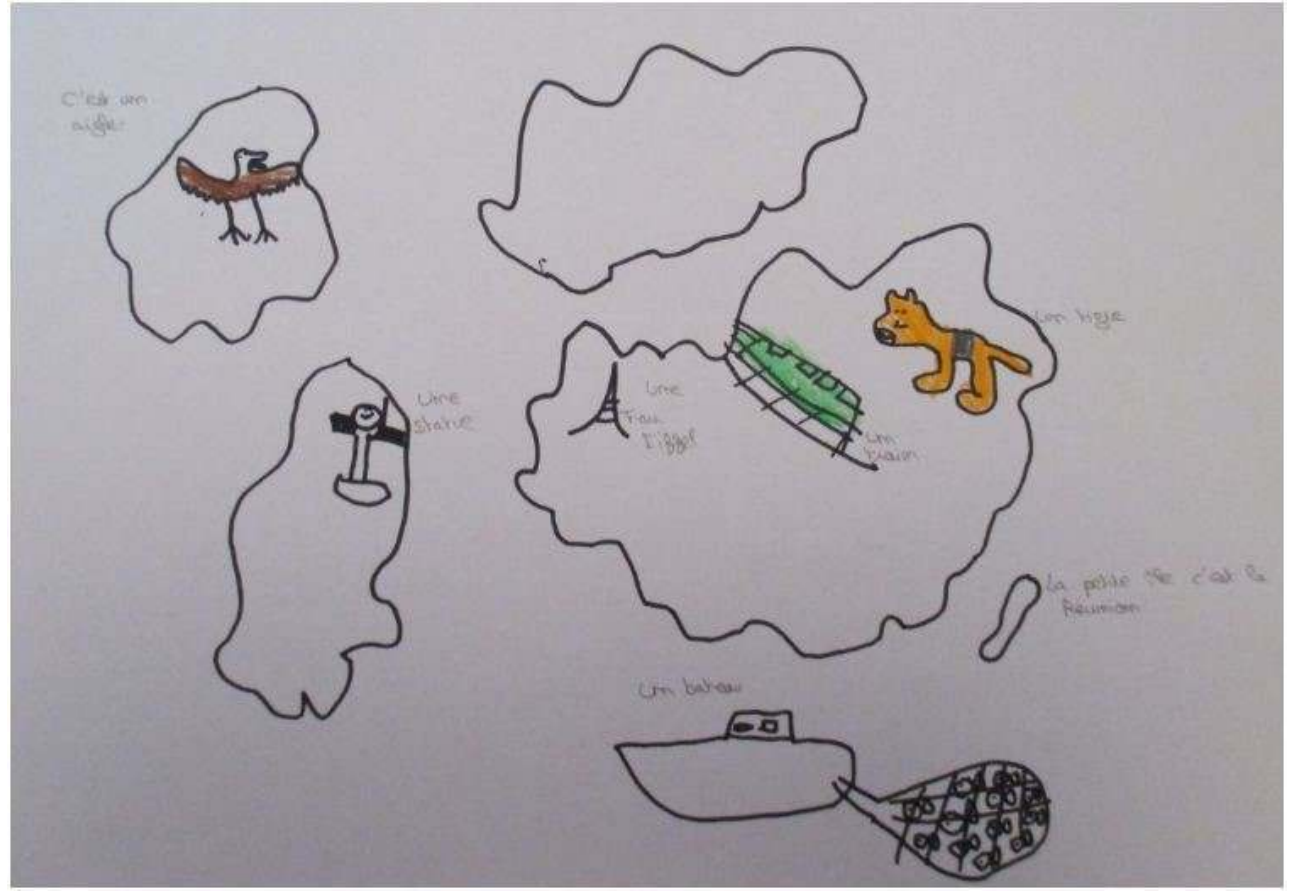

Source : (c) A.-C. Ott, 2014

La carte de Raphaël* ( 5 ans, classe $C^{\prime}$ ) présentée en figure 3 est par ailleurs très inspirée du planisphère présent dans la salle de classe et auquel l'institutrice faisait souvent référence (notamment pour situer les lieux de l'action des albums ou documentaires lus à l'école). Ce qui montre que l'espace scolaire confronte aussi les enfants à ces échelles, par 
exemple au moyen des affichages ou d'un globe, même si elles ne sont pas directement au centre des pratiques d'enseignement.

De même, les élèves de cycle 3 ont pu faire preuve de savoirs géographiques étendus sur l'Europe. Par exemple, sur la question des différences entre l'Europe, l'Union européenne ou la zone euro (comme nous l'avons vu plus haut avec le cas de la Suisse), ou encore des limites de l'Europe. Certains enfants ont montré qu'ils avaient des connaissances précises sur le sujet ${ }^{9}$, proches des savoirs " académiques ", alors même que le sujet n'aurait pas été abordé à l'école. Les exemples de la Russie et de la Turquie ont ainsi été évoqués plusieurs fois par les enfants eux-mêmes :

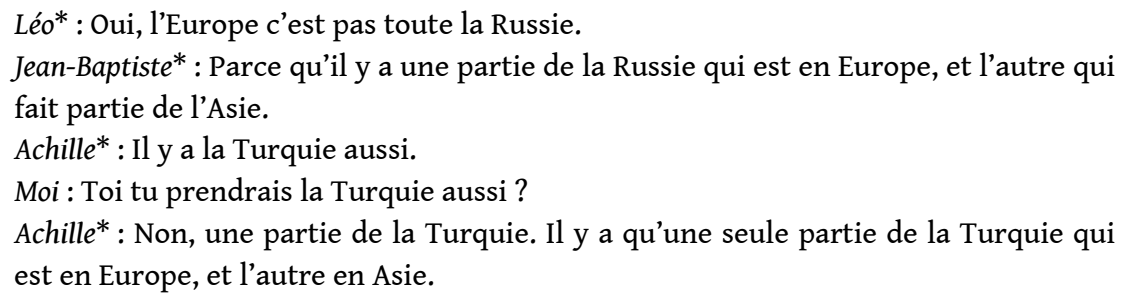

Dès la maternelle, certains enfants avaient des connaissances très précises sur certains sujets, que ce soit sur la localisation de certains pays, sur la provenance de certaines espèces animales ou végétales (un garçon de 5 ans a appris à ses camarades que la pomme Pink Lady venait d'Australie, et que c'est donc pour ça qu'il appréciait particulièrement ce pays), ou encore sur la présence de tel ou tel monument.

\section{Des imaginaires géographiques enfantins différenciés}

Les imaginaires géographiques des enfants rencontrés ne s'arrêtaient donc pas à la frontière du quartier, de la ville, ou du pays, mais ils restaient par contre bien différenciés. La construction des représentations et pratiques spatiales enfantine ne peut effectivement se comprendre que par l'analyse de différents facteurs d'influence, qui forment un système et qu'il faut appréhender dans leurs interactions. Si les travaux de Jean Piaget insistaient particulièrement sur l'importance de l'âge (Piaget, 1926), les recherches plus récentes (Danic, Delalande, Rayou, 2006) et mes propres travaux de terrain tendent à faire ressortir l'impact d'autres facteurs sociaux, au premier rang desquels le milieu social des enfants (mais il ne faut pas non plus négliger les origines ou le genre). Selon les capitaux culturel, social et économique de leur famille, les enfants n'ont pas un accès égal aux savoirs et aux expériences, ce qui joue sur leurs pratiques du Monde mais également sur leurs représentations de ce dernier, de ses espaces et sociétés. Tout d'abord, les enfants ont un accès inégal aux connaissances et aux savoirs, tous n'ont pas la même possibilité de voyager, d'acheter des livres, des globes, des cartes illustrées ou autres objets, mais des inégalités s'observent également au niveau de la transmission des savoirs et de l'exploitation qui est faite par les familles des pratiques et des savoirs.

La mère d'Alice*, professeur des écoles, m'a par exemple expliqué en entretien que lors du voyage familial en Suède, il était important pour elle de faire les conversions des couronnes suédoises en euro avec ses filles pour qu'elles puissent en pratique se rendre compte des différences entre les pays d'Europe. Un voyage en Grèce a quant à lui été organisé à l'occasion du dixième anniversaire d'Alice et a été largement préparé avec les enfants. Alice a d'ailleurs tenu à m'apporter et à me montrer le carnet de bord, qu'elle a entièrement fait elle-même, en racontant le contenu de ses journées de voyage et en 
cherchant quelques documents, comme la carte, qu'elle a placée en page de couverture et qu'elle a complétée et légendée (figure 6) :

Figure 6. Un exemple de valorisation de certaines pratiques touristiques : le carnet de bord d'Alice (10 ans, classe $\left.B^{\prime}\right)$.
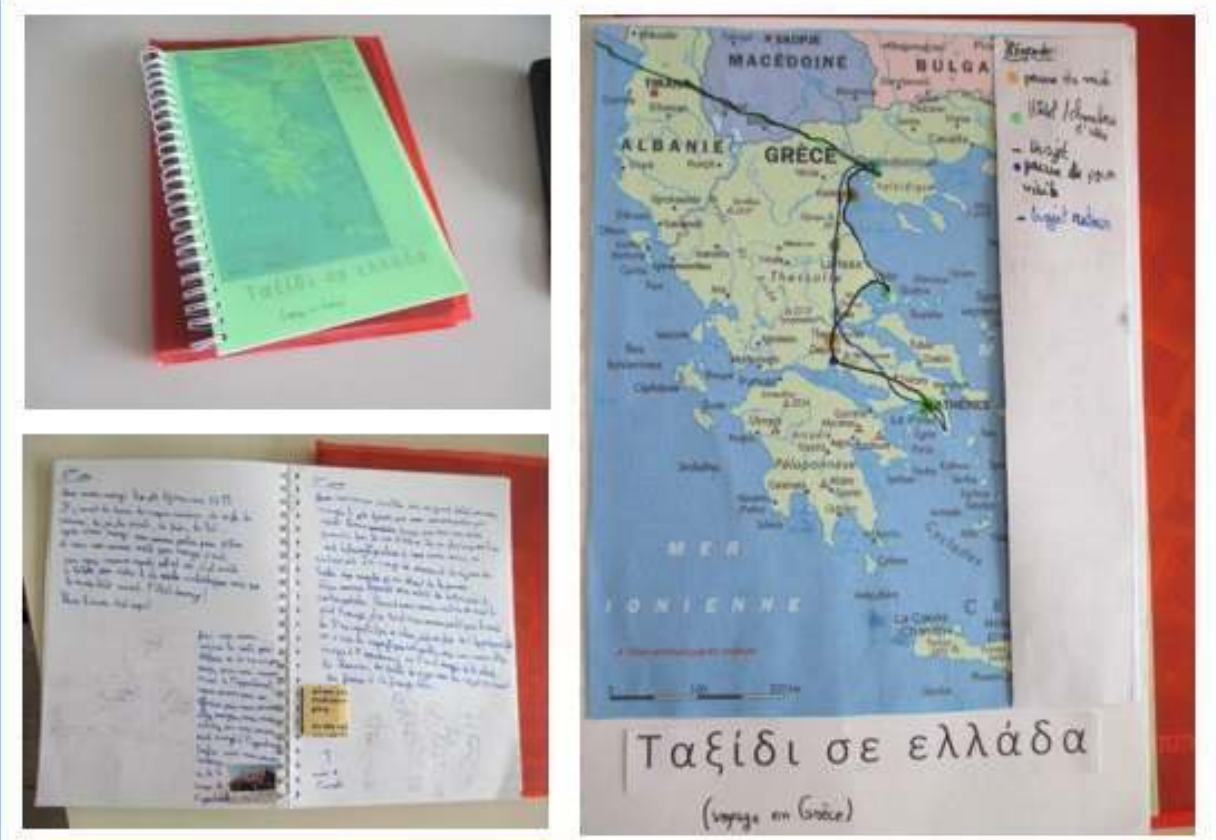

On voit alors que ce ne sont "pas seulement les fréquences des départs, mais aussi les usages sociaux des voyages qui séparent les groupes sociaux» (Wagner, 2007). La pratique touristique s'accompagne effectivement dans les classes supérieures et chez les personnes au capital intellectuel important d'une valorisation du voyage par la production de «savoir-faire et de savoir-être » et est utilisé comme un outil éducatif puissant (ibid.).

De telles expériences ont une influence certaine et durable sur les imaginaires géographiques des enfants. Le rôle de l'Ecole, autre grande structure socialisatrice de long terme a semblé, lui, moins fondamental quant aux représentations spatiales des enfants, qui restaient également fortement marquées par des évènements et expériences ponctuels, comme le résume le schéma qui suit (figure 7) ${ }^{10}$. 
Figure 7. Schéma synthétisant le système d'influences à l'œuvre dans la construction des imaginaires géographiques des enfants rencontrés.

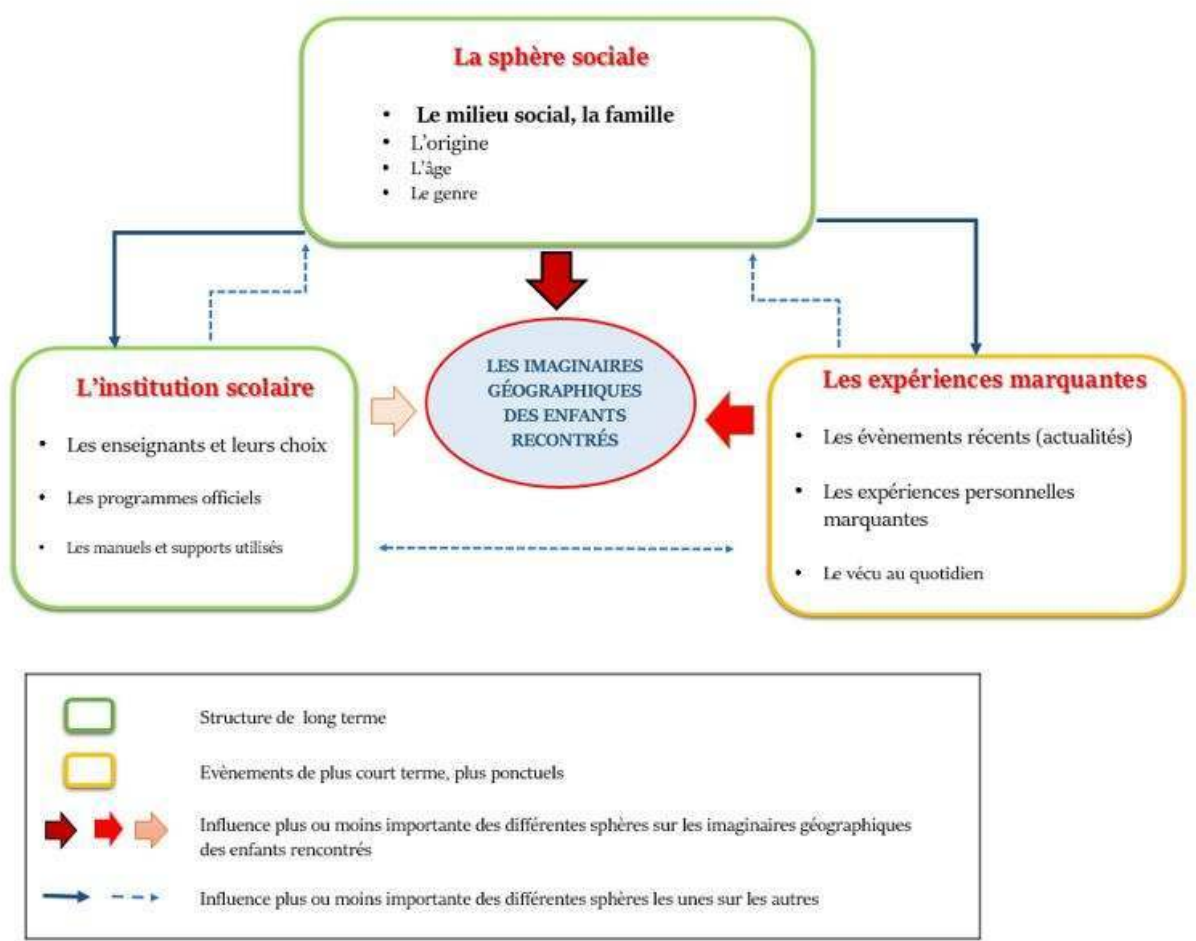

Source : (c) A-C Ott, 2015

\section{Des pratiques et représentations des territoires entre dépolitisation institutionnelle et repolitisation enfantine}

On constate donc un décalage entre le repli de l'enseignement primaire de la géographie sur des espaces de grande échelle et les représentations spatiales des enfants qui s'étendent du local au global, auquel ils sont déjà confrontés. Derrière cette différence, on retrouve un enjeu politique : du côté des institutions, la dépolitisation de la géographie scolaire semble être la règle, et la concentration sur les espaces locaux et nationaux, et sur leurs aspects les plus physiques, économiques ou fonctionnels, permet de ne pas aborder des questions qui paraissent trop appartenir au domaine du politique ${ }^{11}$. Alors que les représentations spatiales des enfants indiquent quant à elles que ces derniers ont un réel sens politique et social.

\section{Un enseignement de la géographie fortement dépolitisé}

La lecture des directives nationales et des travaux de didacticiens ou de chercheurs en sciences politiques permet d'affirmer qu'on fait face à une large dépolitisation de l'enseignement de la géographie. Comme le note Nicole Allieu-Mary, l'enseignement de la géographie, notamment à l'école primaire, mais également au collège et au lycée, correspond surtout à "une vulgate qui expose des savoirs positifs [...] et dénués de toute 
connotation politique » (Allieu-Mary, 2009). On peut par exemple noter que les programmes ne se présentent jamais sous forme de questions (Fontanabona, 2012). Le traitement du problème des limites de l'Europe par exemple illustre bien ce point: les élèves et enseignants ne sont pas appelés à questionner ces limites, mais il faut que le «maître évoque les différentes limites de l'Europe habituellement retenues " (Programme de 2002). Dans les programmes suivants, cette question des limites de l'Europe semble avoir disparu, et il s'agit davantage de savoir placer des pays et quelques villes de l'Union européenne mais pas d'aborder les aspects politiques de cette institution ${ }^{12}$. La dépolitisation de l'enseignement de l'Europe est donc impulsée par les programmes et par les orientations nationales, mais elle serait également due à la peur de la majorité des enseignants d'aborder des sujets trop polémiques. C'est ce qu'a notamment remarqué Géraldine Bozec :

«Les instituteurs évitent toutefois largement de discuter en classe des enjeux qui font l'objet de controverses politiques bien visibles dans le débat public et/ou n'en présentent aux enfants que les aspects les plus factuels ou les plus consensuels » (Bozec, 2010, p. 182).

L'Éducation nationale semble en effet particulièrement réticente à l'idée d'aborder à l'école des sujets perçus comme trop politiques (Allieu-Mary, 2009; Bozec, 2010; Throssell, 2010 ; Fontanabona, 2012). Les enseignants quant à eux peuvent craindre les réactions de certains parents d'élèves, qui n'apprécieraient pas telle ou telle prise de position ou le fait que des questions pouvant prêter à débat soient abordées avec leurs enfants. Katharine Throssell, à la suite d'Annick Percheron, évoque ainsi «la réticence des parents français à penser leur enfants comme un être politique, et une tendance à vouloir le protéger d'un monde adulte et corrompu, en maintenant l'image de l'innocence politique " (Throssell, 2009, p. 65).

Sur le terrain, les enseignants interrogés n'ont toutefois pas affirmé éviter volontairement certains sujets pour des raisons politiques ou par peur de créer de la polémique. Si des thématiques étaient ouvertement évincées (notamment sur l'Europe, ses limites ou ses institutions), c'était selon eux pour des raisons de complexité ou de choix à effectuer, comme nous l'avons vu plus haut. Par ailleurs, aucun d'entre eux n'a exprimé directement la volonté d'aborder des thèmes pour des raisons politiques ou militantes lors des entretiens.

Il est toutefois vrai que les professeurs de primaires rencontrés abordaient tout de même certaines questions sociétales, à travers leur exploration de l'espace local notamment. Il s'agissait principalement de questions environnementales et des comportements civiques à respecter : le projet «L'aventure citoyenne » évoqué plus haut insiste par exemple sur l'apprentissage du « respect des autres et de la planète ». L'échelle locale semblait donc être un espace privilégié pour aborder des questions plus politisées, bien que relativement consensuelles (respect de l'environnement, droits des enfants, comportements civiques, etc.). Nous allons toutefois voir que les enfants, eux, n'hésitaient pas à «repolitiser » leurs rapports aux espaces, et ce y compris à petite échelle. 


\section{Mais un rapport aux espaces (notamment de petite échelle) repolitisé par les enfants}

$$
44
$$
d'émettre des jugements de valeurs sur les espaces qu'ils aimaient ou qu'ils n'aimaient pas. Et cela s'est vérifié en primaire : les enfants de la première école m’ont par exemple demandé si on ne pouvait pas faire une activité où ils auraient à classer en deux colonnes les pays d'Europe qu'ils aimaient et ceux qu'ils n'aimaient pas, alors que ce n'était pas prévu au départ. Ce classement, cette hiérarchisation de pays a par ailleurs pu mettre en lumière des attitudes racistes, comme le montre le dialogue ci-dessous entre des filles de CM1 de la classe $\mathrm{A}^{\prime}$ :

Bunna* [en regardant avec insistance Daniéla ] : J'aime pas la Roumanie.

Danièla* : Pourquoi ? J'aime pas la Maroc.

Bunna* $^{*}$ Mais je suis pas Marocaine!

Danièla* [à une camarade]: Elle habite où elle?

Lila* : Je déteste la Roumanie!

Bunna* : Je déteste la Maroc, Algérie et Tunisie !

Lila : Pourquoi?

Danièla : Elles sont méchants. Surtout les daronnes. [...]

Bunna: La France, c'est là où je suis née.

Danièla : Pourquoi tu mens?! [Rires]

Sorya : Mais si je suis née là-bas ! [...]

Bunna: Je suis née en France, mais c'est juste mon pays d'origine. C'est ma mère qui est née là-bas. Et j'ai mis l'Espagne, parce que j'aime bien parler l'Espagnol et aussi parce que j'aime Violetta. J'aime pas la Roumanie, parce que les Roumanies ils volent des trucs, même dans Cyprien ${ }^{13}$ ça a parlé !

Danièla : Ah tu connais Cyprien?! Les Roms, c'est pas des voleurs hein.

En outre, beaucoup de sujets «politiques» qui sont délibérément évités dans les programmes, par les professeurs et dans les manuels, ont tout de même été spontanément abordés par les enfants. Certains élèves ont également abordé la question du droit d'ingérence de l'Europe, sans pour autant que le sujet soit évoqué en ces termes : l'implication de l'Europe et des Etats européens dans des affaires et des conflits ayant lieu ailleurs dans le Monde a été abordé par Yannis* (classe C'), qui était souvent le premier à mettre cette question à l'ordre du jour durant les différentes activités. Ainsi, durant le brainstorming en classe entière, le jeune garçon a pris la parole pour affirmer que :

«L'Europe elle s'occupe en Afrique de guerres qui sont pas les siennes [...] Elle s'occupe de toutes les guerres qui se passent, elle fournit des armes... ». 
Ces thématiques soulignent ainsi l'implication des enfants dans le champ du politique.

S'intéresser aussi bien à «l'amont de l'acte éducatif » et de l'enseignement primaire de la géographie qu'à « son aval » (Pingué, 2004) permet de mettre en lumière un décalage entre des représentations institutionnelles centrées sur les espaces de grande échelle et des représentations enfantines qui s'étendent du local au global et qui tendent à "repolitiser» certaines questions sociales et géographiques. Ainsi, il semblerait que l'analyse des représentations et pratiques spatiales puisse agir comme révélateurs du sens politique et social des enfants, souvent ignoré voire nié (Throssell, 2009, 2011). S'étant intéressé à la construction du politique chez les enfants, le sociologue Robert Connell montre par ailleurs que c'est par les territoires et leur identification que se forme initialement le rapport des enfants au politique. L'objectif de cet article était donc également de montrer l'apport possible des géographes à une question, encore trop peu étudiée, qui reste pour l'instant largement l'apanage des sociologues ou des spécialistes de sciences politiques.

\section{BIBLIOGRAPHIE}

AITKEN S., HERMANN T. (1997), « Gender, power and crib geography: transitional spaces », Gender, Place and Culture, 4, pp. 63-88.

ALLIEU-MARY N. (2009), « Enseigner l'Europe aujourd'hui, c'est enseigner un objet incertain », Les cafés pédagogiques.net.

ANSELL N. (2009), « Childhood and the politics of scale : descaling children's geographies? ", Progress in Human Geography, 33, 2, pp. 190-209.

BEAUGUITTE L., DIDELON-LOISEAU C. (2013), «L'Europe vue d'ici et d'ailleurs », L'Espace politique, 19.

BOZEC G. (2010), « L'Europe au tableau noir. Comment les instituteurs français enseignent-ils l'Union européenne aujourd'hui ?", Politique européenne, 30, pp. 153-186.

BRENNETOT A., MENDIBIL D. \& ROSEMBERG M. (2011), Europe representations in textbooks, HALarchives-ouvertes.fr, $61 \mathrm{p}$.

BRENNETOT A., ROSEMBERG M. (2013), « Géographie de l'Europe et de la construction européenne. Les images de l'Europe et de l'Union européenne dans quelques manuels de géographie contemporains ", L'Espace politique, 19.

CONNELL R.W. (1971), The Child's construction of politics, Melbourne, Melbourne University Press, $251 \mathrm{p}$.

DANIC I., DELALANDE J. \& RAYON P. (2006), Enquêter auprès d'enfants et de jeunes-Objets, méthodes et terrains de recherche en sciences sociales, Rennes, Presses Universitaires de Rennes, $216 \mathrm{p}$.

FONTANABONA J. (2012), « Originalité de quelques savoirs d'une géographie scolaire de l'Europe ", Didageo - Le blog de la didactique de la géographie, http://

didageo.blogspot.be/2012_03_01_archive.html. 
GRATALOUP C. (2009), L'invention des continents, Paris, Larousse, 224 p.

GRATALOUP C. (2011), « La fausse neutralité des continents », Revue internationale et stratégique, 82, 2.

HOLT L. (dir.) (2011), Geographies of children, youth and families - An international perspective, London, Routledge, 296 p.

LEHMAN-FRISCH S., VIVET J. (2011), « Géographies des enfants et des jeunes », Carnets de géographes, 3.

PHILO C. (2000), «'The corner-stones of my world' : editorial introduction to special issue on spaces of childhood », Childhood, 7, pp. 243-256.

PIAGET J. (1926), La Représentation du monde chez l'enfant, Paris, Presses universitaires de France, $424 \mathrm{p}$.

PINGUE D. (2004), « Note de lecture : Patrick Garcia et Jean Leduc ", L'enseignement de l'histoire en France de l'Ancien Régime à nos jours, Paris, Armand Colin, «Collection U », 2003, 320 p. », Cahiers d'histoire, Revue d'histoire critique, 93.

TERRAIL J-P. (2016), Pour une école de l'exigence intellectuelle. Changer de paradigme pédagogique, Paris, La Dispute, $141 \mathrm{p}$.

THROSSELL K. (2009), « Tous les enfants de ma classe votent Ségolène », Agora débats/jeunesses, 51, pp. 65-78.

THROSSELL K. (2010), « One thing leads to another : European and National identities in French school children », Politique européenne, 30, pp. 131-152.

VALENTINE G., HOLLOWAY S. (2001), « A window on the wider world ? Rural children's use of information and communication technologies ", Journal of rural studies, 17, pp. 383-394.

VALENTINE G., HOLLOWAY S. (2002), « Cyberkids ? Exploring children's identities and social networks in on-line and off-line worlds ", Annals of the Association of American Geographers, 92, 2, pp. 302-319.

WAGNER A.-C. (2007), « La place du voyage dans la formation des élites », Actes de la Recherche en Sciences Sociales, 170 , pp. 58-65.

\section{NOTES}

1. Je me suis d'abord rendue en 2014 dans trois écoles maternelles (et plus précisément dans trois classes de grande section) de Mulhouse qui présentent des profils socio-économiques contrastés (une école avec des élèves issus en grande majorité de milieux défavorisés - que l'on nommera Ecole A ; une présentant un profil intermédiaire - l'Ecole B - ; et enfin une école d'un quartier favorisé - l'Ecole C). J'ai fait de même, l'année suivante, dans trois classes de CM1 ou de CM2 (fin de cycle 3), dans des écoles elles aussi différenciées que l'on appellera A', B', C'. Les écoles C et C' se trouvent dans l'IRIS Rebberg-Nord, quartier le plus bourgeois de Mulhouse, qui présente par exemple des revenus médians par unité de consommation deux fois supérieurs à la moyenne mulhousienne; les écoles B et B' sont situées respectivement dans l'IRIS Rebberg Sud-Ouest et Haut-Poirier Ouest, les revenus médians y sont légèrement supérieurs à la moyenne de la ville. Enfin, l'école A se situe dans une zone urbaine sensible du nord de la ville, dans l'IRIS Bourtzwiller Sud-Ouest, et l'école A' dans un IRIS central, Fonderie Sud: les deux quartiers présentent toutefois des revenus médians par unité de consommation ou un taux de chômage 
plus bas que la moyenne, ainsi qu'une très faible part de cadres et professions intellectuelle supérieure ou de professions intermédiaires.

2. À part le brainstorming avec les CM1-CM2 qui s'est déroulé en classe entière, toutes les activités ont été réalisées avec des enfants en petits groupes, hors de la salle de classe, de manière à ce que les jeunes enquêtés soient le plus à l'aise possible.

3. Les programmes nationaux français pour les écoles maternelles et élémentaires ne changent pas tous les ans. Ces dernières années, un programme a été promulgué en 2002 et un autre en 2008, qui avait donc toujours cours lors de mon terrain. Des circulaires avec des compléments apportés au programme ont toutefois été publiées en 2012. Enfin, un nouveau programme était prévu pour 2015-2016.C'est donc sur les deux programmes de 2002 et 2008, sur les compléments de 2012 et sur les premières directives de 2015 que je m'appuie ici.

4. La classe de sixième, première année du collège, a été intégrée à ce cycle depuis 2015-2016.

5. Nom donné à l'espace transfrontalier partagé par l'Allemagne, la France et la Suisse.

6. Par souci de préserver l'anonymat des enfants, les prénoms ont été modifiés.

7. Cette tendance est pointée du doigt par les chercheurs qui critiquent les nouveaux paradigmes de la "pédagogie rénovée " parfois qualifiée de pédagogie de "l'adaptation au manque " (Terrail, 2016). Selon eux, alors qu'il avait pour vocation d'aider les élèves les plus en difficulté, ce paradigme qui s'est imposé depuis les années 1970 conduirait en fait à accroître les inégalités scolaires, la pédagogie par l'exemple, par le jeu, sans réelle explicitation des concepts et des notions, ne permettant pas à la plupart des enfants issus des classes populaires de s'approprier vraiment les savoirs (ibid.).

8. Notamment par le biais d'un questionnaire administré aux parents d'élèves.

9. Il ne s'agit pas ici de parler en termes de «bonne » ou de «mauvaise » réponse, la thématique des limites de l'Europe étant très compliquée, loin d'être neutre, et bien plus politique que «scientifique " (Grataloup, 2009, 2011).

10. Ce travail est une première étape d'une recherche en cours : il s'agira de confirmer (ou d'infirmer) ces résultats à partir d'analyses supplémentaires, qui mêleront approches qualitative et quantitative.

11. Ce que j'entends ici par politique concerne à la fois la politique, à savoir ce qui concerne les É tats, les partis, les hommes politiques et leurs décisions, mais également le politique au sens plus large, c'est-à-dire ce qui est aux prises avec les rapports de pouvoir et les divisions du monde social.

12. On peut alors s'interroger sur le risque d'une véritable perte de sens induite par le fait que les élèves doivent apprendre des localisations ou des limites sans avoir d'informations sur ce qui a entraîné leur production, c'est-à-dire notamment des causes historiques et politiques.

13. Un jeune homme qui possède une chaîne célèbre de vidéos sur Youtube.

\section{RÉSUMÉS}

À partir de mes travaux de terrain dans des écoles maternelles et primaires alsaciennes, je souhaite interroger les différences qui existent entre les espaces (re)présentés aux enfants par la géographie scolaire et institutionnelle et ceux qui peuplent les imaginaires géographiques enfantins. L'analyse des programmes scolaires et des manuels utilisés en France, ainsi que les entretiens menés auprès des enseignants mettent en lumière une omniprésence des espaces de 
grandes échelles. Cependant, les discussions et activités expérimentales menées avec les enfants montrent que ces derniers mobilisent dans leurs imaginaires géographiques, différenciés, des espaces d'échelles très variées, qui vont du (micro)-local au global. Derrière ce décalage entre les représentations spatiales produites pour les enfants et celles produites par les enfants, on retrouve un enjeu politique. Du côté des institutions, la dépolitisation de la géographie scolaire semble être la règle, alors que les représentations spatiales des enfants indiquent que ces derniers ont un réel sens politique et social, et cela s'observe notamment à travers leur mobilisation d'échelles plus petites (le Monde et l'Europe par exemple).

Building on my master's degree research conducted with children from Alsatian nursery and elementary schools, I will question the differences between spatial representations produced for children and those produced by these actors. The analyses of the French Geography curriculum and of the handbooks, as well as he interviews with teachers and parents underline a strong focus on large-scale spaces. However the experimental methods I have developed to access children's spatial representations show that small scales are also important in their geographical imaginaries, which span from local to global. Behind this discrepancy, political issues seem to be at stake. The Institutions present Geography in a very unpolitical way whereas children's representations, and notably the fact that they often refer to small scales, are good indicators of their political and social awareness.

\section{INDEX}

Mots-clés : enfants, représentations et pratiques spatiales, échelles, imaginaires géographiques, institution scolaire, dépolitisation/ « repolitisation »

Keywords : children, spatial representations and practices, scales, geographical imaginaries, educational institution, depolitization/"repolitization"

\section{AUTEUR}

\section{ANNE-CÉCILE OTT}

Université Paris 1 Panthéon-Sorbonne, UMR 8504-Laboratoire Géographie-cités, Anne-

Cecile.Ott@malix.univ-Paris1.fr 